\title{
SEASONAL INFLUENZA VACCINATION OF HEALTHCARE WORKERS: A NARRATIVE REVIEW
}

\section{MICHAŁ JACEK JĘDRZEJEK and AGNIESZKA MASTALERZ-MIGAS}

Wroclaw Medical University, Wrocław, Poland

Department of Family Medicine

\begin{abstract}
Influenza is an acute respiratory disease caused by the influenza virus which often occurs in outbreaks and epidemics worldwide. The World Health Organization recommends annual vaccination of healthcare workers (HCWs) against influenza, because most of them are involved in the direct care of patients with a high risk of influenza-related complications. Given the significance of the disease burden, a targeted literature review was conducted to assess issues related to influenza vaccination among HCWs. The primary aim of this review was to assess the incidence of influenza among medical personnel and healthcare-associated influenza, and to outline the benefits of influenza vaccination for patients and HCWs themselves. Vaccination of HCWs seems to be an important strategy for reducing the transmission of influenza from healthcare personnel to their patients and, therefore, for reducing patient morbidity and mortality, increasing patient safety, and reducing work absenteeism among HCWs. The benefits of influenza vaccination for their patients and for HCWs themselves are addressed in literature, but the evidence is mixed and often of low-quality. Int J Occup Med Environ Health. 2022;35(2):127-39
\end{abstract}

Key words:

infection control, influenza, healthcare facilities, healthcare workers, healthcare personnel, influenza vaccination

\section{INTRODUCTION}

Influenza (flu) is an acute respiratory infection (ARI) of viral etiology, with a potentially severe and fatal course. It mainly attacks the upper respiratory tract and, less frequently, the bronchopulmonary section [1]. An uncomplicated influenza virus infection usually lasts about a week and is characterized by a typically sudden appearance of high fever, myalgia, headache, fatigue, nonproductive (dry) cough, sore throat and rhinitis. Most patients recover within 1-2 weeks, without the need for causal treatment or hospitalization [2]. However, influenza poses a serious threat for children, pregnant women, the elderly, people with chronic diseases (such as lung diseases, diabetes, cardiovascular diseases, cancers), because in those groups the influenza virus infection can lead to, among other things, exacerbation and/or serious complications of basic diseases, influenza-associated pneumonia or secondary bacterial pneumonia and death [3].

Influenza viruses are classified into 3 types:

- type A - as a result of its antigenic variability ("antigenic drifts" or "antigenic shifts," specific for influenza $\mathrm{A}$ virus), is the cause of large epidemics and pandemics, with a possible severe course of infection;

- type B - causes local outbreaks;

- type $\mathrm{C}$ - causes mild infections in children $[1,3]$.

In the countries of the northern hemisphere, there is a seasonal increase in influenza cases between November and the end of March, with a peak in January-March [4]. The World Health Organization (WHO) estimates that up

Received: October 31, 2020. Accepted: September 30, 2021.

Corresponding author: Michał Jacek Jędrzejek, Wroclaw Medical University, Department of Family Medicine, Syrokomli 1, 51-141 Wrocław, Poland (e-mail: michaljedrzejek@gmail.com). 
to $10 \%$ of adults and approx. $25 \%$ of children have influenza, 3-5 million people develop a severe course of infection, and there are approx. 500000 deaths from influenza, every year worldwide [2]. At the same time, the majority of deaths in industrialized countries affect people aged $>65$ years.

Influenza-related diseases pose a significant challenge to public health, and have serious social and economic consequences (direct costs, including treatment-related costs and indirect costs, i.e., costs mainly arising from sickness absenteeism) [5]. For example, according to the U.S. figures, the total costs associated with seasonal influenza are estimated to be around USD 87 billion, of which approx. 10 billion are direct medical costs [6].

Due to rather unspecified flu symptoms, similar to the spectrum of cold symptoms, patients first seek medical care from primary care physicians. It is this professional group of physicians that bears the burden of increased influenza incidence. Moreover, the patients visit them in the symptomatic stage, i.e., in the period of (peak) infectivity $[7,8]$. It is worth noting at this point that the possibility of transmission of the influenza virus may be bi-directional, i.e., there is a valid exposure of medical personnel (healthcare workers [HCWs], healthcare personnel or healthcare professionals [HCPs]) to an influenza virus from an infected patient, and physician-topatient transmission of the virus is also possible $[9,10]$. Many health organizations recommend annual influenza vaccination among HCWs because most of them are involved in the direct care of patients with a high risk of influenza-related complications. Influenza vaccination is considered as a key element of control activities intended to prevent nosocomial (healthcare-associated) influenza transmission and, therefore, to reduce patient morbidity and mortality, to increase patient safety, and to reduce work absenteeism among HCWs [11]. Given the significance of these recommendations, a targeted literature review was conducted to provide a holistic view of issues related to influenza vaccination among medical staff. The primary aims of this review were:

- to assess the incidence of influenza among medical personnel,

- to assess healthcare-associated influenza (HAI),

- to outline the benefits of influenza vaccination for patients,

- to outline the benefits of influenza vaccination for HCWs.

\section{METHODS}

A targeted literature search of PubMed was conducted to assess the issues related to influenza vaccination among medical personnel using the following keywords: "influenza," "influenza vaccination," "healthcare personnel," "healthcare workers," "healthcare facilities," and "infection control." Original peer-reviewed articles in English were used. No restriction was placed on the publication date of studies, and all relevant systematic reviews and meta-analyses were also included. The reference lists of the studies included in this analysis were scanned in order to identify additional relevant papers.

\section{RESULTS}

\section{Influenza and medical staff}

Incidence of influenza among medical personnel

A systematic review and meta-analysis of the incidence of influenza among medical personnel and other healthy adults by Kuster et al. [9] (58 245 participants in total; influenza seasons 1957-2009) suggest that medical personnel faces a higher risk of symptomatic influenza infections (up to 2.5 times) compared to the population of healthy adults working in establishments other than healthcare facilities. The above study review estimates that up to $22 \%$ of HCWs (especially those not vaccinated against influenza) can have influenza every epidemic season. The authors also attempt to explain the higher rates of asymptomatic influenza infections among medi- 
cal personnel in comparison with other healthy non-HCWs, and they hypothesize that those HCWs who are more exposed to influenza infections (admission of/contact with symptomatic patients during the infection season and/or post-vaccination exposure) develop more effective immunity mechanisms that reduce the severity of infection symptoms.

This problem was pointed out already in the 1990s: according to an English study conducted among hospital workers who were found to have serological signs of a past influenza virus infection (23.2\% of the total number of 518 analyzed workers; the epidemic season 1993-1994), 59\% of them did not remember the fact of having had influenza, and $28 \%$ of them did not remember airway infections at all [12]. On the other hand, in a German study (involving 677 participants; the epidemic season 2014-2015), 24\% of hospital staff reported the occurrence of ARI during the infection period ( $83 \%$ reported coughing), with $9 \%$ of the staff reporting a likely influenza infection (defined as fever $\geq 38.5^{\circ} \mathrm{C}$ and a sudden appearance of symptoms) [13]. Interestingly, the above-mentioned study did not demonstrate a statistical relationship between the reported probable influenza infection and immunization status (a possible selection bias). Another interesting report is the analysis of medical staff of the emergency room and the operating room (117 staff members in total) during the influenza $\mathrm{A}(\mathrm{H} 1 \mathrm{N1})$ pdm09 pandemic season, whose results revealed that a total of $29 \%$ of staff members showed seroconversion in anti-hemagglutinin antibodies [14]. Seroconversion concerned $36.8 \%$ of the emergency room staff and $14.6 \%$ of the operating room staff, so the risk of serologically confirmed influenza infection was 3.4 times higher for the emergency room staff. In addition, also in their case, higher (nearly twice as high) antibody titers were observed. About one-third of the seropositive people (35.3\%) did not develop symptoms of influenza-like illness (ILI). The above-mentioned study has re-evaluated the usefulness of the existing prevention measures, such as protective masks and hand hygiene, which have been associated with a reduced risk of serologically confirmed influenza infection.

Presenteeism associated with ILIs among medical personnel Results of various studies directly show that a large group of physicians (even $>75 \%$ [15]) admit that they perform their professional duties while having symptoms of upper respiratory tract infections (the so-called presenteeism) $[11,16,17]$. For example, according to the results of an American survey (involving 1914 HCWs; the influenza season 2014-2015), 41.4\% of the respondents reported being present at work with influenza-like symptoms (a median of 3 days), while pharmacists and physicians were the ones who most commonly reported being present at work when sick $(67.2 \%$ and $63.2 \%$, respectively) [18]. In this way, medical personnel can introduce the influenza virus and perpetuate its transmission, putting patients at risk [11].

\section{Influenza related to healthcare Incidence of influenza related to healthcare}

The phenomenon of HAI is becoming increasingly important in literature. Although hospital influenza epidemics occur in almost all types of wards and have significant consequences for patients and hospitals, the source of infection is often unknown [10]. Given, among other things, the lack of standardized diagnostic methods and, above all, the absence of routine epidemiological surveillance, comprehensive estimates of the occurrence of this phenomenon are impossible. For example, according to the Canadian Nosocomial Infection Surveillance Program (CNISP) of 2006-2012, almost 20\% of laboratoryconfirmed influenza infections among hospitalized adult patients were classified as healthcare-related (HAI was recorded if the symptoms appearing in an infection were equal to or greater than those occurring $96 \mathrm{~h}$ after admission or less than $96 \mathrm{~h}$ if the patient was (re)admitted 

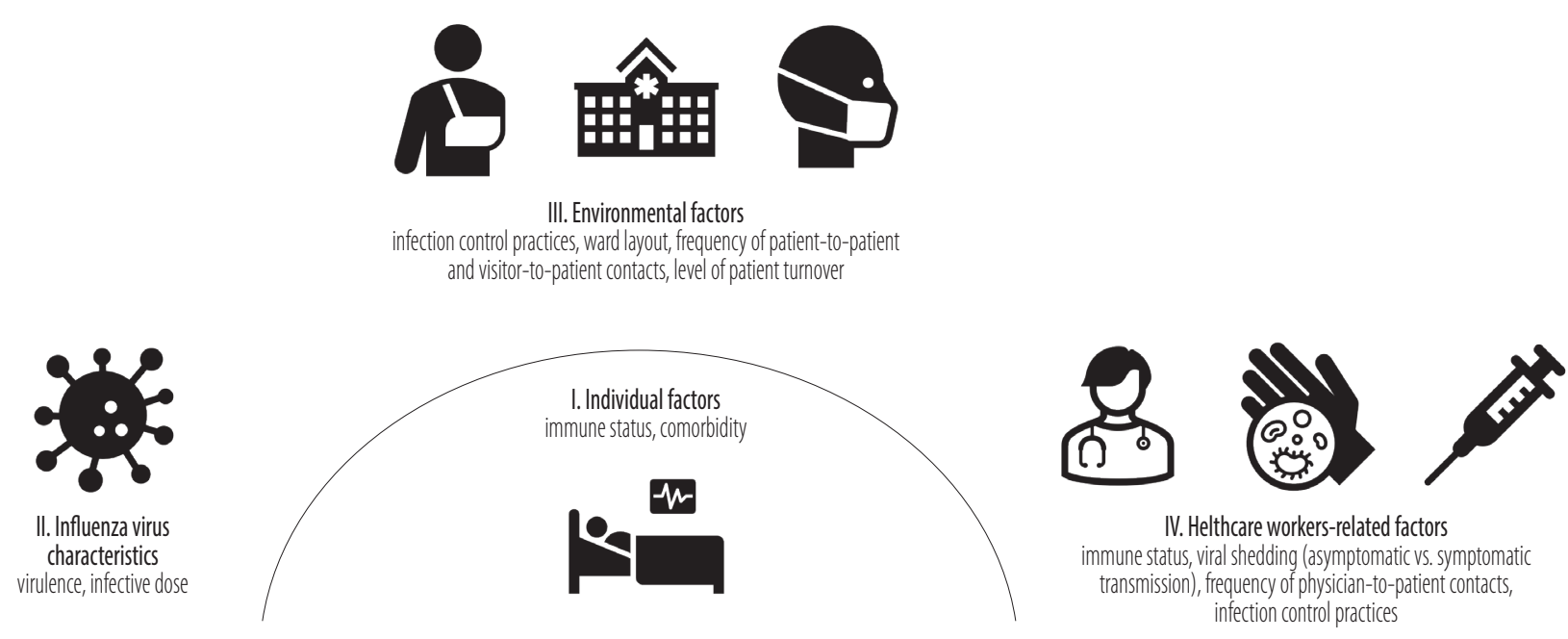

Figure 1. Factors affecting nosocomial influenza infections (authors' own analysis)

after discharge or transfer from another facility) [19]. It is worth noting that systematic laboratory epidemiological surveillance, independent of the definition of a clinical (symptomatic) case, would also allow the recording of asymptomatic cases (the percentage of people with asymptomatic influenza virus infection is estimated at $28-59 \%$ [10]).

Influenza related to healthcare - the role of healthcare personnel Although the published data support the hypothesis that healthcare personnel may serve as a vector for the spread of influenza among hospitalized patients, resulting in a variety of negative effects, including increased costs of hospital care [10], there are debatable data on the impact of HCW vaccination against influenza on reducing the incidence of influenza among patients [20]. The authors draw attention to the complexity of determinants of nosocomial infections (Figure 1).

An interesting observation was described by Pagani et al. [21] during a hospital influenza epidemic (a geriatric care hospital in Switzerland, the infection season 2011-2012). Namely, a genetic analysis demonstrated that multiple community strains of the virus were respon- sible for nosocomial influenza outbreaks, which refuted the hypothesis of outbreaks caused by a single influenza strain transmitted within the healthcare facility. The same authors also noticed that non-vaccinated HCWs could play a key role in perpetuating the outbreaks. The authors pointed to the need for research to estimate the burden of ARIs, and especially influenza, in the HCWs group and to determine their role in the transmission of healthcareassociated respiratory infections $[9,13,20]$. Therefore, it seems to be of value to undertake a cross-sectional and, above all, prospective/longitudinal study to determine the prevalence (detectability) of the microbiological presence of the influenza virus in HCWs during the epidemiological season, and to correlate the findings with the actual vaccination status and clinical symptoms.

\section{Influenza vaccination of medical personnel - benefits for patients}

It is widely believed that vaccinations of medical personnel are beneficial to patients and it is worth noting that over 30 years ago the American Advisory Committee on Immunization Practices (ACIP) recommended annual influenza vaccination for those HCPs who cared for high- 
risk patients and, as such, should be considered as a reservoir of infection, capable of transmitting influenza to patients [15]. Therefore, preventive vaccination against influenza should be viewed not only as part of medical personnel vaccination but also as a practice that has an ethical and moral dimension. It is proposed that the percentage of vaccinated staff should be taken into account as one of the parameters of the safety of medical facilities and quality of medical care [11].

The results of the studies carried out so far show that long-term care facilities (LTCFs) with higher influenza vaccination rates among residents and staff have reported lower influenza incidence [22]. Influenza vaccination of medical personnel can also reduce the overall mortality rate in older patients in LTCFs, although more research and analysis of this relationship are advisable [23-28]. The authors of a comprehensive review dated 2019 believed that there was strong evidence showing a benefit of HCW vaccinations to patients, at least for all-cause mortality [20]. In turn, a study in a hospital with an oncological profile (a 8-year observation) showed directly that an increase in HCW vaccination rates (from 56\% in 2006-2007 to 94\% in 2013-2014) was significantly associated with a decreased percentage of nosocomial influenza infections [29]. A similar effect was observed in a facility in South Carolina in the USA (an increase in HCW vaccination rates from $4 \%$ to $67 \%$ in $1987-2000$ ) [30].

It is worth quoting here interesting results of the analysis of comparing the effectiveness of influenza vaccination of medical personnel and hospital patients, where the adjusted value was $89 \%$ among HCWs and $42 \%$ among patients (twice less effective) [31]. The results may explain the protective effect of vaccinated HCPs on patients for whom influenza vaccination may be less effective (the so-called cocoon strategy). The authors of the British literature review also reported the possible effectiveness of influenza vaccination against laboratory-confirmed influenza among medical personnel at $88 \%$ and healthy adults at approx. 60\% [32].
Vaccination efficacy (VE) against clinically suspected influenza, in accordance with the so-called principle of dilution, was naturally lower in both groups, reaching up to approx. 50\% for the HCWs group and up to approx. 20\% for the group of healthy adults (confirmation of influenza etiology is obtained within a broad range from approx. 23\% [33] to approx. 51\% [34] of ILIs). Despite the above data, the benefits for patients deriving from influenza vaccination of medical personnel are still ambiguous and inconsistent in literature, and are still widely debated [20,32].

\section{Criticism of the mandatory vaccination policy}

The most recent literature also criticizes the policy of mandatory vaccinations among medical personnel (USA, Canada), pointing to the lack of reliable empirical evidence on the real benefits for patients. The strongest criticism is revealed in the paper concerning cluster randomized controlled trials conducted in LTCFs [35]: their results served as an input for developing the rules for mandatory influenza vaccinations among HCWs in the USA. The authors claimed that the provided benefits for patients resulting from HCW vaccination were mathematically impossible (e.g., the results reported did not comply with the principle of dilution) and they estimated that the number needed to vaccinate (NNV) should be at least 6000-32 000 HCWs to prevent 1 patient death [35]. However, the authors of the UK literature review dated 2016, quoting the authors who had reported no benefits or a likely protective effect of healthcare personnel vaccination for patients, ultimately concluded that it might be postulated that vaccinating HCWs with an effective vaccine was very likely to reduce the transmission of influenza onto patients [32].

\section{Influenza vaccination of medical personnel - individual benefits}

The individual vaccination benefits for medical personnel are not so well-documented, although the studies carried out so far have shown a (slight) decrease in 
the number of days of sickness absenteeism [36,37] (by approx. 0.5 days $[32,38]$ ) and a reduction in the risk of influenza virus infection [9,37]. The authors of the 2011 review suggested that the only statistically significant result in the review was that influenza vaccination could, to some extent, reduce the incidence of laboratory-confirmed influenza infection among HCWs [39]. The authors of the UK literature review reported that a NNV to prevent 1 case of influenza among HCWs is around 40 [32]. Other data from the UK study suggest that a $10 \%$ increase in the HCW vaccination rate may be associated with a $10 \%$ decrease in sickness absenteeism [40].

In turn, the authors of the 2019 review concluded that the evidence for most issues related to HCW vaccinations (risk of influenza and transmission of infections from HCWs to patients, the benefits of HCW vaccination) is mixed and often of low-quality [20]. They also indicated that there is significant heterogeneity in the design of existing studies, which makes them difficult to compare. At the same time, they concluded that, regardless of the above limitations, most studies suggest that the vaccination of HCWs against influenza is an important policy for both medical personnel and patients.

\section{DISCUSSION}

The fact that influenza virus infections continue to be a serious phenomenon is confirmed, for example, by the great Spanish influenza (1918), the "Asian" or HongKong pandemics in 1957 and 1968 [1,2], and the recent A(H1N1)pdm09 swine flu pandemic in the 2009-2010 season [3]. Annual preventive vaccination remains a key tool for influenza prevention. In the current era of evidence-based medicine, both HCPs and the general public expect clear evidence of the recommended wide use of influenza vaccination. In recent years, several comprehensive literature reviews have attempted to rigorously analyze various aspects related to influenza vaccination, including among HCWs $[9,20,32,41]$. The wide variety of research designs and a poor quality of most evidence do not allow universal and unambiguous conclusions to be drawn for policy makers or healthcare managers. Therefore, poor implementation and low acceptance of the current recommendations for annual influenza vaccination, both among healthcare personnel and the general population, are hardly surprising [20].

Globally, it is estimated that the HCW influenza vaccination rates range $2-44 \%$ and the recommended optimal influenza vaccination coverage rate for medical personnel to protect patients is approx. 90\% [42]. However, according to Jenkin et al. [20], influenza virus infections are likely to remain among the major methodological challenges for scientists (e.g., the influenza burden on the population and the infectivity of virus strains change every year, also spatially) to obtain high-quality conclusive data on the benefits of influenza vaccination among HCWs.

The current knowledge base needs to be supplemented. It is still unclear whether influenza vaccination may be able to fully block the chain of transmission, or whether it simply reduces the severity of the disease in vaccinated subjects. The differences may lie in the selection or, more precisely, in how the vaccine works. For instance, an inactivated influenza vaccine (IIV) leads to the production of neutralizing serum antibodies (relatively narrow and strain-specific response) while immunization with live attenuated influenza vaccine (LAIV; mucosal administration) results in the production of both serum and mucosal antibodies (a broader and potentially more prolonged response) [43]. A very high efficacy in blocking the horizontal transmission of the influenza virus by LAIV is confirmed by animal studies, although immunization with whole, killed influenza virus reduced the viral load after the challenge and partially reduced the number of secondary transmission cases [44]. Worth noting is the fact that IIVs are commonly used: for instance, they represented $89.6 \%$ of global production of seasonal influenza vaccines in 2019 while LAIV account- 
ed for $5.0 \%$ [45]. The influenza virus triggers a very complex immune response and the role of mucosal immunity is currently the subject of ever more wide-ranging research and debate.

An effective surveillance and control strategy for influenza infection also depends on reliable estimates of the asymptomatic rate and the contribution of asymptomatic individuals to the influenza virus transmission chain. Although microbiological studies have shown that the intensity of influenza virus shedding is directly correlated with the severity of infection symptoms $[7,8,46]$, virus shedding also occurs in asymptomatic individuals. Influenza virus is detectable in the upper respiratory tract of infected individuals up to several days before the onset of symptoms, as well as in individuals without clinical symptoms of influenza or ILI $[8,46]$. For example, in a systematic review and meta-analysis of 55 studies with laboratory-confirmed influenza cases, the frequency of asymptomatic influenza infection ranged 5.2-35.5\% [47], although some authors reported a value of $28-59 \%$ [10]. There is an ongoing debate in literature about the role of these individuals in transmission, i.e., whether virus shedding and spread occurs beyond the nasopharynx if no symptoms are present [11]. In consequence, physical preventive measures based on symptoms only (wearing masks, washing and disinfecting hands, keeping a distance) may not be sufficient, whereas an epidemic may be controlled more effectively through general preventive measures, including influenza vaccinations [8]. Therefore, further studies are needed regarding the impact of influenza vaccination, among other things, on mucosal immunity and the frequency of asymptomatic influenza infections, and on the role of asymptomatic individuals, including medical personnel, in the infection transmission chain.

Nevertheless, so far it can be stated that:

- influenza among HCWs and HAI is a recognized problem;
- HCWs are a professional group that is particularly at risk of continued work during infection;

- there is sufficient data to conclude that influenza vaccines are effective (moderate effectiveness: the meta-analysis of 2013 concerning VE depending on the match between their antigenic composition and circulating strains in a particular influenza epidemic season showed that a trivalent inactivated vaccine; [currently the use of a quadrivalent influenza vaccine is recommended] ensured protection both in mismatches [9 randomized clinical trials: VE 52\%], and matches [8 randomized clinical trials: VE 65\%] of the antigenic composition of formulations among adults [48]) and safe (the authors of Cochrane's literature review did not find any evidence of an association between influenza vaccination and serious adverse events in healthy adults [49]);

- HCWs can participate in the transmission of influenza events;

- there are strong signals that HCW influenza vaccination protects patients, especially with regard to mortality [20].

As other authors further point out, vaccination becomes the primary strategy that will protect patients if HCWs are indeed at a particularly high risk of asymptomatic influenza infection [9]. It appears, therefore, that the above data justify all efforts to increase influenza vaccination rates among HCWs.

\section{The role of occupational physicians}

Interventions that promote vaccination should take into account both the individual beliefs of workers (the health belief model) and the socio-cultural and organizational context of the facilities where they are implemented (the socio-ecological model) [20]. It is also worth remembering that those interventions that are well-established in the context of the health belief model may sometimes encounter (unexpected) resistance arising from socio- 
cultural and organizational contexts in which they are implemented. Moreover, the larger the facility and, thus, the more numerous the staff, the more resources and efforts are required for promotional and educational activities in order to create conducive conditions for vaccination (e.g., a well-planned and organized immunization program, adequate time for performing the vaccination and efforts to ensure availability of vaccines) [50].

At this point, the important role of occupational physicians in promoting influenza vaccination for medical personnel in the workplace comes to the fore. For example, in a 10-year observation in the USA on the effectiveness of interventions to increase influenza vaccination coverage, hospital facilities with staff vaccination rates exceeding $80 \%$ were small rural hospitals or hospitals with limited staff counts (308-2092 people), whereas the lowest vaccination rate was recorded in the largest academic hospital with more than 8700 employees [51]. However, it should be noted that during the last year of observation, $>5300$ employees of the aforementioned academic hospital were vaccinated, and this number was higher than the total number of employees vaccinated in 5 other (smaller) hospitals.

Apart from the organizational context, the individual beliefs and the level of knowledge of occupational physicians themselves may also play an important role for the vaccination rates of medical staff. For example, in an Italian study of 92 occupational physicians (the percentage of those vaccinated against influenza was $46.7 \%$ in the infection season 2014-2015), 23.9\% questioned the efficiency of vaccines and almost $20 \%$ of them incorrectly declared that vaccines may be causatively related to diabetes mellitus or autism [52]. Similarly, German data reported that in a group of 135 occupational physicians, $16.3 \%$ did not deny that vaccination could trigger diseases such as diabetes or autism [53]. Nevertheless, $88.9 \%$ of physicians in the German study were strongly or completely in favor of vaccination, and $98.5 \%$ of them recommended influ- enza vaccination for HCWs [53], whereas in the Italian study $95.6 \%$ described themselves as somewhat favorable toward vaccinations, with $68.5 \%$ being somewhat favorable towards influenza vaccination [52].

The authors conclude that there is ample evidence that greater awareness (higher levels of knowledge) and greater confidence in vaccines increase the willingness to vaccinate which, in the case of occupational physicians, would entail their greater willingness to perform and promote vaccination in the workplace [52]. Therefore, any educational interventions should involve not only medical staff, i.e., employees who are the main recipients of vaccination programs, but also the implementers, i.e., the medical staff who are members of the occupational health team. These interventions should aim to correct potential misconceptions and to shape pro-vaccination attitudes, including towards influenza vaccination, as knowledge and attitudes are important predictors of recommending vaccination.

\section{Medical personnel}

\section{as an authority in health choices in the context of universal influenza vaccination}

The secondary aspect in these considerations is the importance and impact of HCW influenza vaccination on the behavior and attitudes of the general population in the context of widespread influenza vaccination. Despite the proven beneficial effects of influenza vaccination, the vaccination rates in the general population are very variable in different areas of the world. For example, the rate for Poland is extremely low at around 3.5\% (data regarding 2008-2018 [4]). The health choices in the Euro-American cultures are increasingly based on scientific knowledge, and medical personnel, as a group perceived as trustworthy due to their high level of competence and professional experience, can have a positive impact on health behavior. Primary healthcare staff, especially family doctors, should play a central role in preventive interventions, including the promotion of preventive vac- 
cination, as they are perfectly placed to clarify patients' concerns and find ways to explain the benefits of vaccination to them [54]. For example, in a survey conducted in 5 European populations in 2007-2008, advice from a family doctor was the most effective factor (58.6\%) for vaccination in all countries [55].

It is also worth remembering that the advice and care provided by doctors to their patients is also influenced by their personal behavior and pro-health attitudes. Studies confirm that a higher percentage of patients receive an influenza vaccine if their doctor has also been vaccinated [56]. However, since some HCWs are not vaccinated, there is a real concern and risk that hesitant HCWs may spread the concern about vaccines to the general population, less frequently recommend vaccination to their patients, as well as reduce confidence in vaccination and, consequently, patient acceptance [54]. This is, therefore, another argument in favor of the efforts to increase the influenza vaccination rates among HCWs.

\section{Influenza vaccination and COVID-19}

It should be remembered that, in the era of the COVID-19 pandemic, infection by 1 pathogen does not rule out the possibility of co-infection by another one, including the influenza virus [57]. While influenza vaccination has variable and moderate efficacy, given the current epidemiological situation, it seems appropriate to make every effort to reduce the burden of influenza virus-induced infections on the healthcare system by carrying out universal (population-based) influenza vaccinations. Experts from the Centers for Disease Control and Prevention (CDC) believe that influenza vaccination in the era of the COVID-19 pandemic should not only decrease the incidence of influenza, but also help protect potentially limited healthcare resources [58]. In turn, other authors calculate that assuming a 50\% effectiveness of the influenza vaccine and an average value of Basic Reproduction Number (R0) of the influenza virus at 1.5, herd immunity of the population would be achieved if at least $66.6 \%$ of the population were vaccinated [59]. Given the fact that influenza vaccination can reduce the percentage of influenza virus infections among healthcare personnel $[9,39]$ and, to some extent, lower sickness absenteeism [32,3638], CDC experts believe it is also recommended to protect as many HCWs as possible against influenza before the coming infection season [58].

The current epidemiological situation has highlighted the need to optimize universal influenza vaccination. The authors indicate that, in the face of a dramatically increased demand for influenza vaccines, the technological barrier of manufacturing efficiency, regulated, among other things, by the availability of substrates, has become particularly visible [60]. When talking about production limitations, the authors primarily highlight factors such as dependence on the availability of embryonated chicken eggs since both seasonal IIV and LAIV are eggbased $(84.5 \%$ of global production capacity; cell culture is an alternative substrate for production, accounting for the remaining 15.5\%) [45]. Even in periods of typical demand for vaccine preparations, production may be compromised, e.g., due to avian influenza and the resulting reduced supply of chicken eggs [60]. Another limitation is the relatively time-consuming production period, i.e., annual vaccine formulation forecasts are prepared months before the onset of the influenza season in order to include all stages of vaccine production and distribution, which poses the risk of poor matching between vaccine strains and strains circulating in a particular epidemiological season $[45,60]$.

In view of the possible limited supply of vaccine preparations, it is reasonable to select specific groups from the general population, as well as from the HCW population, to receive influenza vaccination as a priority. In particular, these would be individuals with an increased risk of severe course of influenza and/or development of complications (i.e., children, pregnant women, the elderly, 
people with chronic diseases, e.g., lung diseases, diabetes, cardiovascular diseases, cancers) and all frontline HCPs who directly provide services to patients at a high risk of influenza-related complications [58].

\section{CONCLUSIONS}

The available data show that influenza, including HAI, is a recognized problem and that influenza vaccines are safe and effective. Influenza vaccination can reduce influenza virus infections among HCWs and the number of sick leave days taken by workers. The benefits of HCW influenza vaccination for patients are still inconsistent and widely discussed. Given the safety, effectiveness and other possible benefits of influenza vaccines, all efforts to increase influenza vaccination rates among HCWs are reasonable, especially in the era of the COVID-19 pandemic.

\section{REFERENCES}

1. Orthomyxoviruses and influenza. In: Collier L, Oxford J, editors. Human Virology. New York: Oxford University Press; 2006. p. 87-95.

2. WHO. Influenza fact sheet: Overview. Wkly Epidemiol Rec. 2003;78:77-80.

3. Moghadami M. A Narrative Review of Influenza: A Seasonal and Pandemic Disease. Iran J Med Sci. 2017;42(1):2-13.

4. Byambasuren S, Paradowska-Stankiewicz I, Brydak LB. Epidemic Influenza Seasons from 2008 to 2018 in Poland: A Focused Review of Virological Characteristics. AdvExp Med Biol. 2020;1251:115-21, https://doi.org/10.1007/5584_2019_462.

5. Peasah SK, Azziz-Baumgartner E, Breese J, Meltzer MI, Widdowson M-A. Influenza cost and cost-effectiveness studies globally - a review. Vaccine. 2013;31(46):5339-48, https:// doi.org/10.1016/j.vaccine.2013.09.013.

6. Molinari N-AM, Ortega-Sanchez IR, Messonnier ML, Thompson WW, Wortley PM, Weintraub E, et al. The annual impact of seasonal influenza in the US: measuring disease burden and costs. Vaccine. 2007;25(27):5086-96, https://doi. org/10.1016/j.vaccine.2007.03.046.
7. Carrat F, Vergu E, Ferguson NM, Lemaitre M, Cauchemez S, Leach S, et al. Time Lines of Infection and Disease in Human Influenza: A Review of Volunteer Challenge Studies. Am J Epidemiol. 2008;167(7):775-85, https://doi.org/10. 1093/aje/kwm375.

8. Ip DKM, Lau LLH, Chan K-H, Fang VJ, Leung GM, Peiris MJS, et al. The Dynamic Relationship Between Clinical Symptomatology and Viral Shedding in Naturally Acquired Seasonal and Pandemic Influenza Virus Infections. Clin Infect Dis. 2016;62(4):431-7, https://doi.org/10.1093/cid/civ909.

9. Kuster SP, Shah PS, Coleman BL, Lam P-P, Tong A, Wormsbecker $\mathrm{A}$, et al. Incidence of influenza in healthy adults and healthcare workers: a systematic review and meta-analysis. PLoS One. 2011;6(10):e26239, https://doi.org/10.1371/jour nal.pone.0026239.

10. Voirin N, Barret B, Metzger M-H, Vanhems P. Hospitalacquired influenza: a synthesis using the Outbreak Reports and Intervention Studies of Nosocomial Infection (ORION) statement. J Hosp Infect. 2009;71(1):1-14, https:/doi.org/ 10.1016/j.jhin.2008.08.013.

11. Perl TM, Talbot TR. Universal Influenza Vaccination Among Healthcare Personnel: Yes We Should. Open Forum Infect Dis. 2019;6(4):ofz096, https://doi.org/10.1093/ofid/ofz096.

12. Elder AG, O’Donnell B, McCruden EAB, Symington IS, Carman WF. Incidence and recall of influenza in a cohort of Glasgow healthcare workers during the 1993-4 epidemic: results of serum testing and questionnaire. BMJ. 1996;313 (7067):1241-2, https://doi.org/10.1136/bmj.313.7067.1241.

13. Hagemeister MH, Stock NK, Ludwig T, Heuschmann P, Vogel U. Self-reported influenza vaccination rates and attitudes towards vaccination among health care workers: results of a survey in a German university hospital. Public Health. 2018;154:102-9, https://doi.org/10.1016/j.puhe.2017.10.027.

14. Sandoval C, Barrera A, Ferrés M, Cerda J, Retamal J, GarcíaSastre A, et al. Infection in Health Personnel with High and Low Levels of Exposure in a Hospital Setting during the H1N1 2009 Influenza A Pandemic. PLoS One. 2016;11(1):e0147271, https://doi.org/10.1371/journal.pone.0147271. 
15. Weingarten S, Riedinger M, Bolton LB, Miles P, Ault M. Barriers to influenza vaccine acceptance. A survey of physicians and nurses. Am J Infect Control. 1989;17(4):202-7.

16. Orr P. Influenza vaccination for health care workers: A duty of care. Can J Infect Dis. 2000;11(5):225-6.

17. Widera E, Chang A, Chen HL. Presenteeism: A Public Health Hazard. J Gen Intern Med. 2010;25(11):1244-7, https://doi. org/10.1007/s11606-010-1422-x.

18. Chiu S, Black CL, Yue X, Greby SM, Laney AS, Campbell AP, et al. Working with influenza-like illness: Presenteeism among US health care personnel during the 2014-2015 influenza season. Am J Infect Control. 2017;45(11):1254-8, https://doi.org/10.1016/j.ajic.2017.04.008.

19. Taylor G, Mitchell R, McGeer A, Frenette C, Suh KN, Wong A, et al. Healthcare-Associated Influenza in Canadian Hospitals from 2006 to 2012. Infect Control Hosp Epidemiol. 2014; 35(2):169-75, https://doi.org/10.1086/674858.

20. Jenkin DC, Mahgoub H, Morales KF, Lambach P, NguyenVan-Tam JS. A rapid evidence appraisal of influenza vaccination in health workers: An important policy in an area of imperfect evidence. Vaccine X. 2019;2:100036, https://doi. $\operatorname{org} / 10.1016 /$ j.jvacx.2019.100036.

21. Pagani L, Thomas Y, Huttner B, Sauvan V, Notaridis G, Kaiser L, et al. Transmission and Effect of Multiple Clusters of Seasonal Influenza in a Swiss Geriatric Hospital. J Am Geriatr Soc. 2015;63(4):739-44, https://doi.org/10.1111/ jgs.13339.

22. Stevenson CG, McArthur MA, Naus M, Abraham E, McGeer AJ. Prevention of influenza and pneumococcal pneumonia in Canadian long-term care facilities: how are we doing? CMAJ. 2001;164(10):1413-9.

23. Carman WF, Elder AG, Wallace LA, McAulay K, Walker A, Murray GD, et al. Effects of influenza vaccination of healthcare workers on mortality of elderly people in long-term care: a randomised controlled trial. Lancet. 2000;355(9198):93-7, https://doi.org/10.1016/S0140-6736(99)05190-9.

24. Ahmed F, Lindley MC, Allred N, Weinbaum CM, Grohskopf L. Effect of Influenza Vaccination of Healthcare Personnel on
Morbidity and Mortality Among Patients: Systematic Review and Grading of Evidence. Clin Infect Dis. 2014;58(1):50-7, https://doi.org/10.1093/cid/cit580.

25. Potter J, Stott DJ, Roberts MA, Elder AG, O’Donnell B, Knight PV, et al. Influenza vaccination of health care workers in long-term-care hospitals reduces the mortality of elderly patients. J Infect Dis. 1997;175(1):1-6, https://doi. org/10.1093/infdis/175.1.1.

26. Hayward AC, Harling R, Wetten S, Johnson AM, Munro S, Smedley J, et al. Effectiveness of an influenza vaccine programme for care home staff to prevent death, morbidity, and health service use among residents: cluster randomised controlled trial. BMJ. 2006;333(7581):1241, https://doi.org/ 10.1136/bmj.39010.581354.55.

27. Lemaitre M, Meret T, Rothan-Tondeur M, Belmin J, Lejonc J-L, Luquel L, et al. Effect of influenza vaccination of nursing home staff on mortality of residents: a cluster-randomized trial. J Am Geriatr Soc. 2009;57(9):1580-6, https:// doi.org/10.1111/j.1532-5415.2009.02402.x.

28. Thomas RE, Jefferson T, Lasserson TJ. Influenza vaccination for healthcare workers who work with the elderly. Cochrane Database Syst Rev. 2010;2:CD005187, https:/doi. org/10.1002/14651858.CD005187.pub3.

29. Frenzel E, Chemaly RF, Ariza-Heredia E, Jiang Y, Shah DP, Thomas G, et al. Association of increased influenza vaccination in health care workers with a reduction in nosocomial influenza infections in cancer patients. Am J Infect Control. 2016; 44(9):1016-21, https://doi.org/10.1016/j.ajic.2016.03.024.

30. Salgado CD, Giannetta ET, Hayden FG, Farr BM. Preventing Nosocomial Influenza by Improving the Vaccine Acceptance Rate of Clinicians. Infect Control Hosp Epidemiol. 2004;25(11):923-8, https://doi.org/10.1086/502321.

31. Vanhems P, Baghdadi Y, Roche S, Bénet T, Regis C, Lina B, et al. Influenza vaccine effectiveness among healthcare workers in comparison to hospitalized patients: A 2004-2009 case-test, negative-control, prospective study. Hum Vaccin Immunother. 2016;12(2):485-90, https://doi.org/10.1080/ 21645515.2015 .1079677$. 
32. Kliner M, Keenan A, Sinclair D, Ghebrehewet S, Garner P. Influenza vaccination for healthcare workers in the UK: appraisal of systematic reviews and policy options. BMJ Open. 2016;6(9):e012149, https://doi.org/10.1136/bmjopen-2016012149 .

33. Chittaganpitch M, Waicharoen S, Yingyong T, Praphasiri P, Sangkitporn S, Olsen SJ, et al. Viral etiologies of influenzalike illness and severe acute respiratory infections in Thailand. Influenza Other Respi Viruses. 2018;12(4):482-9, https://doi.org/10.1111/irv.12554.

34. Cinemre H, Karacer C, Yücel M, Ögüülü A, Cinemre FB, Tamer A, et al. Viral etiology in adult influenza-like illness/ acute respiratory infection and predictivity of $\mathrm{C}$-reactive protein. J Infect Dev Ctries. 2016;10(7):741-6, https://doi. org/10.3855/jidc.6939.

35. De Serres G, Skowronski DM, Ward BJ, Gardam M, Lemieux C, Yassi A, et al. Influenza Vaccination of Healthcare Workers: Critical Analysis of the Evidence for Patient Benefit Underpinning Policies of Enforcement. PLoS One. 2017;12(1):e0163586, https://doi.org/10.1371/journal.pone.0163586.

36. Frederick J, Brown AC, Cummings DA, Gaydos CA, Gibert CL, Gorse GJ, et al. Protecting Healthcare Personnel in Outpatient Settings: The Influence of Mandatory Versus Nonmandatory Influenza Vaccination Policies on Workplace Absenteeism During Multiple Respiratory Virus Seasons. Infect Control Hosp Epidemiol. 2018;39(4):452-61, https://doi.org/10.1017/ice.2018.9.

37. Wilde JA, McMillan JA, Serwint J, Butta J, O’Riordan MA, Steinhoff MC. Effectiveness of influenza vaccine in health care professionals: a randomized trial. JAMA. 1999;281(10): 908-13, https://doi.org/10.1001/jama.281.10.908.

38. Saxén H, Virtanen M. Randomized, placebo-controlled double blind study on the efficacy of influenza immunization on absenteeism of health care workers. Pediatr Infect Dis J. 1999;18(9):779-83, https://doi.org/10.1097/00006454199909000-00007.

39. $\mathrm{Ng}$ ANM, Lai CKY. Effectiveness of seasonal influenza vaccination in healthcare workers: a systematic review. J Hosp
Infect. 2011;79(4):279-86, https://doi.org/10.1016/j.jhin. 2011.08.004.

40. Pereira M, Williams S, Restrick L, Cullinan P, Hopkinson NS, London Respiratory Network. Healthcare worker influenza vaccination and sickness absence - an ecological study. Clin Med (Lond). 2017;17(6):484-9, https://doi.org/10.7861/clinmedicine.17-6-484.

41. Dini G, Toletone A, Sticchi L, Orsi A, Bragazzi NL, Durando P. Influenza vaccination in healthcare workers: A comprehensive critical appraisal of the literature. Hum Vaccin Immunother. 2018;14(3):772-89, https://doi.org/10.1080/ 21645515.2017.1348442.

42. Maltezou HC, Tsakris A. Vaccination of health-care workers against influenza: our obligation to protect patients. Influenza Other Respi Viruses. 2011;5(6):382-8, https://doi.org/ 10.1111/j.1750-2659.2011.00240.x.

43. Krammer F. The human antibody response to influenza A virus infection and vaccination. Nat Rev Immunol. 2019;19: 383-97, https://doi.org/10.1038/s41577-019-0143-6.

44. Lowen AC, Steel J, Mubareka S, Carnero E, García-Sastre A, Palese P. Blocking Interhost Transmission of Influenza Virus by Vaccination in the Guinea Pig Model. J Virol. 2009;83(7):2803-18, https://doi.org/10.1128/JVI.02424-08.

45. Sparrow E, Wood JG, Chadwick C, Newall AT, Torvaldsen S, Moen A, et al. Global production capacity of seasonal and pandemic influenza vaccines in 2019. Vaccine. 2021;39(3): 512-20, https://doi.org/10.1016/j.vaccine.2020.12.018.

46. Lau LLH, Cowling BJ, Fang VJ, Chan K-H, Lau EHY, Lipsitch $\mathrm{M}$, et al. Viral Shedding and Clinical Illness in Naturally Acquired Influenza Virus Infections. J Infect Dis. 2010; 201(10):1509-16, https://doi.org/10.1086/652241.

47. Furuya-Kanamori L, Cox M, Milinovich GJ, Magalhaes RJS, Mackay IM, Yakob L. Heterogeneous and Dynamic Prevalence of Asymptomatic Influenza Virus Infections. Emerg Infect Dis. 2016;22(6):1052-6, https://doi.org/10.3201/eid2 206.151080 .

48. Tricco AC, Chit A, Soobiah C, Hallett D, Meier G, Chen MH, et al. Comparing influenza vaccine efficacy against mis- 
matched and matched strains: a systematic review and meta-analysis. BMC Med. 2013;11(1):153, https://doi.org/ 10.1186/1741-7015-11-153.

49. Demicheli V, Jefferson T, Ferroni E, Rivetti A, Di Pietrantonj C. Vaccines for preventing influenza in healthy adults. Cochrane Database Syst Rev. 2018;2(2):CD001269, https:// doi.org/10.1002/14651858.CD001269.pub6.

50. To KW, Lai A, Lee KCK, Koh D, Lee SS. Increasing the coverage of influenza vaccination in healthcare workers: review of challenges and solutions. J Hosp Infect. 2016;94:133-42, https://doi.org/10.1016/j.jhin.2016.07.003.

51. Ajenjo MC, Woeltje KF, Babcock HM, Gemeinhart N, Jones M, Fraser VJ. Influenza Vaccination among Healthcare Workers: Ten-Year Experience of a Large Healthcare Organization. Infect Control Hosp Epidemiol. 2010;31:233-40, https://doi.org/10.1086/650449.

52. Ricco M, Cattani S, Casagranda F, Gualerzi G, Signorelli C. Knowledge, attitudes, beliefs and practices of Occupational Physicians towards seasonal influenza vaccination: a crosssectional study from North-Eastern Italy. J Prev Med Hyg. 2017;58(2):E141-54.

53. Betsch C, Wicker S. Personal attitudes and misconceptions, not official recommendations guide occupational physicians' vaccination decisions. Vaccine. 2014;32:4478-84, https:// doi.org/10.1016/j.vaccine.2014.06.046.

54. European Centre for Disease Prevention and Control [Internet]. Stockholm: The Centre; 2015 [cited 2020 Oct 30].
Vaccine hesitancy among healthcare workers and their patients in Europe. Available from: https://www.ecdc.europa. eu/en/publications-data/vaccine-hesitancy-among-health care-workers-and-their-patients-europe.

55. Blank PR, Schwenkglenks M, Szucs TD. Disparities in influenza vaccination coverage rates by target group in five European countries: trends over seven consecutive seasons. Infection. 2009;37(5):390-400, https://doi.org/10.1007/s15 010-009-8467-y.

56. Frank E, Dresner Y, Shani M, Vinker S. The association between physicians' and patients' preventive health practices. CMAJ. 2013;185(8):649-53, https://doi.org/10.1503/ cmaj.121028.

57. Belongia EA, Osterholm MT. COVID-19 and flu, a perfect storm. Science. 2020;368(6496):1163, https://doi.org/10.11 26/science.abd2220.

58. Centers for Disease Control and Prevention [Internet]. The Centers; 2021 [cited 2021 Feb 10]. Interim Guidance for Routine and Influenza Immunization Services During the COVID-19 Pandemic. Available from: https://www.cdc. gov/vaccines/pandemic-guidance/index.html.

59. Grech V, Borg M. Influenza vaccination in the COVID-19 era. Early Hum Dev. 2020;148:105116, https://doi.org/10.10 16/j.earlhumdev.2020.105116.

60. Houser K, Subbarao K. Influenza Vaccines: Challenges and Solutions. Cell Host Microbe. 2015;17(3):295-300, https:// doi.org/10.1016/j.chom.2015.02.012.

This work is available in Open Access model and licensed under a Creative Commons Attribution-NonCommercial 3.0 Poland License - http://creativecommons.org/ licenses/by-nc/3.0/pl/deed.en. 